\title{
Nanoscale
}

D) Check for updates

Cite this: DOI: $10.1039 / \mathrm{c} 8 \mathrm{nr} 05787 \mathrm{~g}$

\section{Optimizing the yield of A-polar GaAs nanowires to achieve defect-free zinc blende structure and enhanced optical functionality $\dagger$}

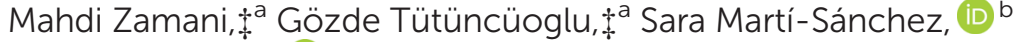 \\ Luca Francaviglia, (D) ${ }^{a}$ Lucas Güniat, ${ }^{a}$ Lea Ghisalberti, ${ }^{a}$ Heidi Potts, ${ }^{a}$ Martin Friedl, ${ }^{a}$ \\ Edoardo Markov, ${ }^{a}$ Wonjong Kim, ${ }^{a}$ Jean-Baptiste Leran, ${ }^{a}$ Vladimir G. Dubrovskii, ${ }^{c}$ \\ Jordi Arbiol (DD ${ }^{\text {b,d }}$ and Anna Fontcuberta i Morral (D) *a
}

\begin{abstract}
Compound semiconductors exhibit an intrinsic polarity, as a consequence of the ionicity of their bonds. Nanowires grow mostly along the (111) direction for energetic reasons. Arsenide and phosphide nanowires grow along (111)B, implying a group $V$ termination of the (111) bilayers. Polarity engineering provides an additional pathway to modulate the structural and optical properties of semiconductor nanowires. In this work, we demonstrate for the first time the growth of Ga-assisted GaAs nanowires with (111)A-polarity, with a yield of up to $\sim 50 \%$. This goal is achieved by employing highly Ga-rich conditions which enable proper engineering of the energies of A and B-polar surfaces. We also show that A-polarity growth suppresses the stacking disorder along the growth axis. This results in improved optical properties, including the formation of AlGaAs quantum dots with two orders or magnitude higher brightness. Overall, this work provides new grounds for the engineering of nanowire growth directions, crystal quality and optical functionality.
\end{abstract}

Received 18th July 2018

Accepted 24th August 2018

DOI: $10.1039 / c 8 n r 05787 g$

rsc.li/nanoscale originating from the different electron affinity of the composing elements. The sign and magnitude of this finite electrical field depends on the crystal direction. Compound semiconductor NWs tend to grow in the $<111>$ directions. (111) surfaces are composed by bi-layers of III-V dumbbells with an intrinsic electric field normal to the surface. When the surface is terminated by a group III or V element, it is named (111)A and (111)B, respectively. Recent developments in electron microscopy allow for a relatively straightforward determination of the polarity of NW growth direction. ${ }^{15-17}$ NWs tend to grow with a prevalent polarity, depending on the material. For example, $\mathrm{Ga}(\mathrm{N}, \mathrm{P}, \mathrm{As}), \operatorname{In}(\mathrm{N}, \mathrm{P}, \mathrm{As}, \mathrm{Sb})$ and $\mathrm{Zn}(\mathrm{S}, \mathrm{Se}, \mathrm{Te})$ tend to grow along the $<111>\mathrm{B}$ direction, while $\mathrm{ZnO}$ shows mostly $<111>$ A-polarity. ${ }^{15-21}$

Recently, it has been shown that the structural properties of compound semiconductor NWs can vary significantly when their growth direction and polarity are modified. ${ }^{22}$ In bulk, GaAs exhibits zinc blende (ZB) structure, while the presence of polytypism and wurtzite phase (WZ) in (111)B-polar GaAs NWs is well documented. ${ }^{23-25}$ Few groups have assessed the engineering of crystal growth polarity by modifying the contact angle of the VLS droplet. Yuan et al. demonstrated for the first time that modifying the droplet wetting and surface energy allowed for the switching of the polarity of Au-catalyzed GaAs NWs from the usual B to A. ${ }^{26}$ According to their study, A-polar 
NWs are obtained with higher contact angles, which they explained by the higher surface energy of A-polar surfaces. The presence of rotational twins ${ }^{27}$ and polytypism typically observed in B-polar NWs was completely suppressed in A-polar NWs. This was an important finding as it opened the path toward growth of defect-free GaAs NWs. Another recent study showed similar results on Sn-seeded GaSb NWs. ${ }^{28}$ In this case, the A-polar NWs were defect-free, in contrast to the B-polar NWs which had spaced stacking defects appearing in the $<111>$ A direction. The presence of these defects resulted in zigzag-shaped sidewalls in B-polar NWs.

In this work, we demonstrate for the first time that GaAs NWs can be reliably grown in the $<111>$ A direction by the Ga-assisted VLS method. This shows that catalyst for growth of A-polar GaAs NWs does not need to be restricted to gold. This observation is significant, because gold-assisted growth is not compatible with the existing semiconductor industry. We provide a parametric study on the occurrence of the two polarities as a function of $\mathrm{Ga}$ and $\mathrm{As}_{4}$ fluxes and substrate. The detailed study of the crystal structure of NWs having different polarities is performed by aberration-corrected high-angle annular dark field scanning transmission electron microscopy (AC-HAADF-STEM). The relation between the nanowire polarity and the optical properties have been studied by low temperature cathodoluminescense (CL).

\section{Experimental details}

First, the GaAs (100) substrates were etched in HF to remove the native oxide. The silicon oxide layers were obtained by spin coating a solution of Hydrogen Silsesquioxane (HSQ) and Methyl Isobutyl Ketone (MIBK). The spin-coated layers were annealed at $300{ }^{\circ} \mathrm{C}$ for 10 minutes. The oxide thicknesses used for all the samples were around $3.3 \mathrm{~nm}$. Two additional annealing steps at $150{ }^{\circ} \mathrm{C}$ and $300^{\circ} \mathrm{C}$ for two hours each were performed inside the MBE chamber. The initial chamber pressure for all the growths was less than $5 \times 10^{-10}$ Torr. For the sample discussed in Fig. 6, the growth conditions for the NW core were: the V/III ratio of 1.9, 2D growth rate of $0.24 \AA_{\mathrm{s}^{-1}}$, at a substrate temperature of $640{ }^{\circ} \mathrm{C}$. The growth conditions for the shell were: As flux of $9.3 \times 10^{-6}$ mbar, $2 \mathrm{D}$ growth rate of $0.7 \AA \mathrm{s}^{-1}$, substrate temperature of $450{ }^{\circ} \mathrm{C}$.

The TEM samples were prepared by scraping a TEM grid on an as-grown sample. ${ }^{29}$ Atomic resolution HAADF-STEM was performed in a probe corrected FEI Titan 60-300 keV microscope operated at $300 \mathrm{keV}$. TEM images given in Fig. 5 were obtained with an FEI Talos machine operating at $200 \mathrm{keV}$.

The CL measurements were performed using an Attolight Rosa 4634 SEM-CL microscope at the Interdisciplinary Center for Electron Microscopy (CIME), EPFL. The measurements reported in Fig. 5(h) and 6(a) were performed using a beam accelerated to $5 \mathrm{keV}$, while for the measurements in Fig. 6(i) and $5(\mathrm{j})$ this value was $8 \mathrm{keV}$. For all the measurements, the acquisition time of each spectra was $0.1 \mathrm{~s}$ and the beam current was on the order of $1 \mathrm{nA}$.

\section{Results and discussion}

Polarity in compound semiconductor NWs can be determined in an unambiguous manner by identifying the atomic columns in the material, typically using AC-HAADF-STEM techniques. $^{22}$ These techniques are precise at the atomic level but incompatible with a fast statistical assessment on a large ensemble of NWs. Alternatively, the NW growth polarity can be determined by their orientation with respect to the epi-substrate, which in our case is $\operatorname{GaAs}(100)$. Fig. 1 represents the atomic arrangement of NWs oriented along the $<111>\mathrm{A}$ and $<111>$ B with respect to such substrate. In the insets, we provide the details of atomic order when looking along the $[-110]$ and [110] zone axes, respectively. We have assessed the

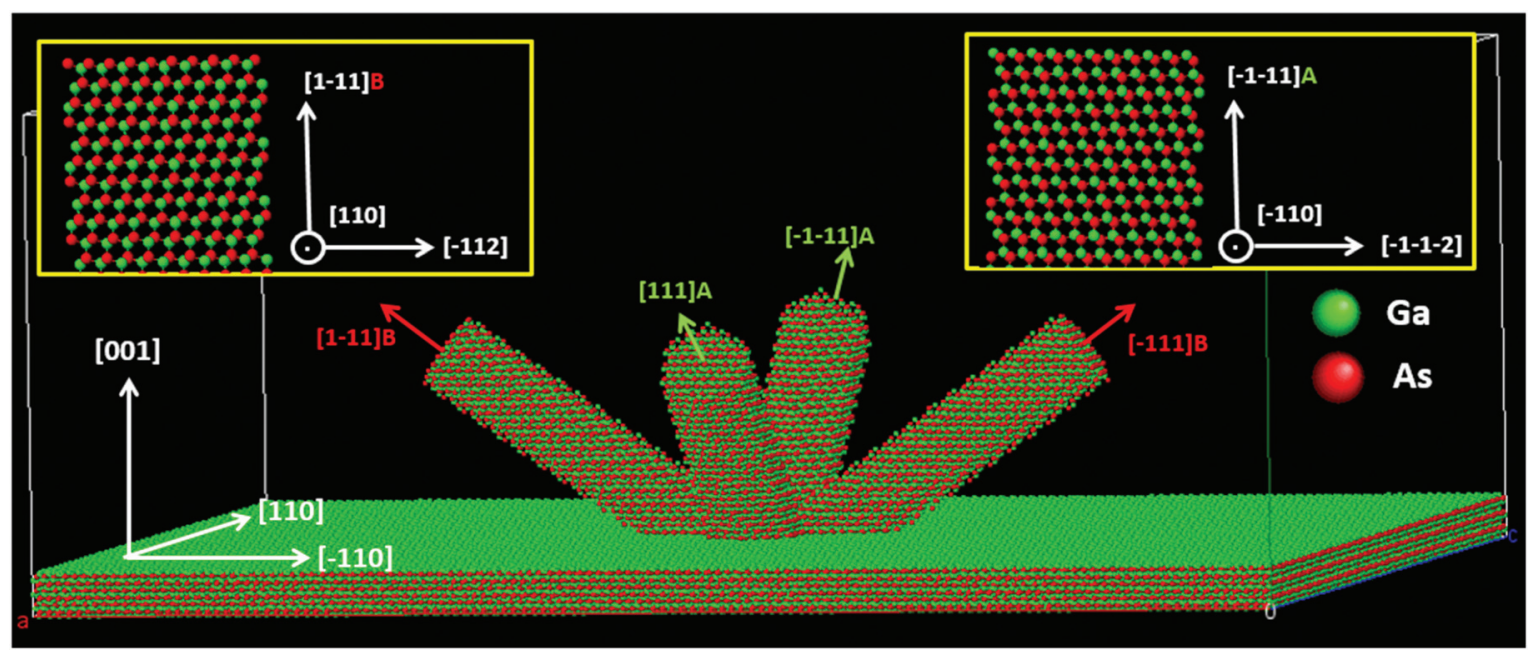

Fig. 1 Three-dimensional atomic model of GaAs NWs oriented along the $<111>B$ and $<111>A$ directions on a GaAs(100) substrate. The insets zoom in the atomic ordering in the $[110]$ and $[-110]$ zone axis, respectively, for $B$ and A-polar NWs. 
polarity of NWs in ensembles by correlating their orientation with the crystalline axes of the substrate using top view SEM micrographs.

Fig. 2(a) shows a representative top-view scanning electron microscope (SEM) image of one of the grown samples. Here, the NW projections appear oriented along the two perpendicular directions, which correspond to the $<111>$ A and $<111>$ B directions. A closer look at this image reveals the presence of NWs crawling on the surface. Similar structures were previously observed in the VLS growth of NWs. ${ }^{30}$ A low V/III ratio yields the formation of big droplets, which can easily be unpinned from the top of the structures in the early stages of growth and fall down onto the substrate. The subsequent growth originating from such droplets will result in the formation of crawling structures. ${ }^{31}$ Increased oxide thickness or hole density might prevent the appearance of crawling objects. $^{32-35}$ Fig. 2(b) and (c) show the cross-sectional SEM images of another sample in the two perpendicular cleaving directions. One can distinguish the two NW populations also in this configuration. As expected, the angle of the NWs with respect to the substrate normal is $54.5^{\circ}$.

We have studied the ratio between number of the $\mathrm{B}$ and A-polar NWs as a function of the growth parameters. For this, we have kept the substrate temperature at $640{ }^{\circ} \mathrm{C}$ and modified the V/III ratio and the Ga flux. Statistics of the population of A-polar NWs obtained from examining the top-view SEM images is shown in Fig. 2(d). We observe an increase in the population of A-polar NWs when reducing the V/III ratio. This effect will be discussed shortly. Unexpectedly, the Ga flux itself also affects the $\mathrm{A} / \mathrm{B}$ ratio, with an optimum value being around 0.21-0.24 $\AA^{-1}$. It was not possible to distinguish the two polarities in the range of grey data points due to the shorter lengths of the nanowires.
From Fig. 2(b), we see that the fraction of A-polar NWs can be tuned to 0.57 for $\mathrm{V} / \mathrm{III}$ ratios of around 2 and Ga rates of 0.21-0.24 $\AA^{-1}$. We note that the growth conditions leading to the highest yield of A-polar NWs on GaAs(100) substrates do not work on (111)A substrates. Instead, NWs crawling on the substrate surface was mostly observed on (111)A under similar conditions. We attribute this result to the different wetting properties of the Ga droplet on (100), (111)B and (111)A surfaces.

These results indicate a competition between the nucleation of A and B-polar GaAs NWs on GaAs(100) substrates, where the A-polarity is preferred at low V/III ratios. A quantitative description of the nucleation probabilities for NWs with different parameters under different $\mathrm{Ga}$ and $\mathrm{As}_{4}$ fluxes requires a separate study and will be presented elsewhere. Here we just mention that the liquid droplet catalyzing the VLS growth of any NWs should wet the growth front but not the NW sidewalls. $^{26,28,36}$ In general, (111)A facets are rarely seen as the growth front in a nanowire. One reason could be that their surface energy would be higher than for (111)B and hence their formation would be energetically and geometrically suppressed in favor of the (111)B facets. ${ }^{31,37,38}$ The (111) surface energy as well as the nucleation probability of GaAs is also affected by kinetic or thermodynamic factors such as chemical potential in the liquid phase. ${ }^{2,42}$ It is very possible that a reduction in the As concentration under lower As fluxes causes the effective energies of $\mathrm{A}$ and $\mathrm{B}$ surfaces to decrease and increase, respectively. This results in increasing the wetting chance of an A-polar surface with respect to a B-polar surface. For this reason, lowering the V/III ratio could increase the probability of wetting A-polar surfaces and hence growing A-polar NWs. We note that the previous attempts to grow A-polar GaAs and GaSb NWs (gold and tin-catalyzed, respect-
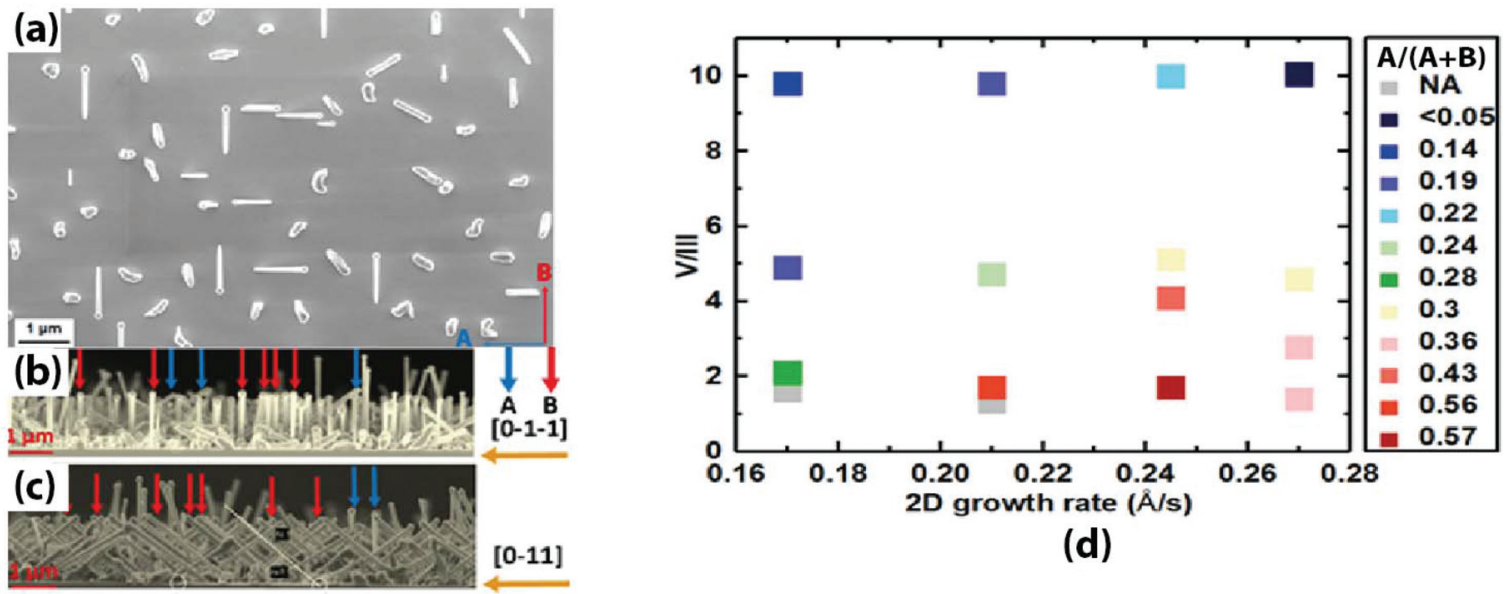

(d)

Fig. 2 (a) Top view SEM image of a sample grown with a Ga rate of $0.21 \AA \mathrm{s}^{-1}$ and a V/III ratio of 1.7. The colored arrows indicate the projections corresponding to $<111>A$ and $<111>B$ directions. (b) and (c) Cross-sectional SEM images of a sample grown with a Ga rate of $0.60 \AA \mathrm{s}^{-1}$ and a V/III ratio of 2.9, cleaved along the two $\langle 100>$ directions the colored arrows indicate the NW polarity (A or B) according to their orientation with respect to the (100) GaAs substrate. (d) Fraction of A-polar NWs as a function of V/III ratio and the 2D equivalent Ga growth rate. There is a clear increase in A-polar NW fraction when the V/III ratio is reduced. In addition, the Ga flux itself also affects the fraction of A-polar NWs, with a clear maximum in the range of $0.21-0.24 \AA \mathrm{s}^{-1}$. 
ively $)^{26,28}$ used a similar strategy for increasing the wettability of A-polar surfaces, although without changing the V/III ratio to achieve this goal. The coexistence of NWs of different polarities can be explained by the fact that the modification in the energy of A-polar surfaces might not be enough to make the wetting of the B-polar surfaces completely unfavorable. ${ }^{26,28}$ The highest yield of A-polar NWs corresponds to extremely low $\mathrm{As}_{4}$ fluxes. Further decrease of the $\mathrm{As}_{4}$ input cannot be achieved in a reproducible manner because the lowest flux is already close to the background pressure under our growth conditions. Hence, it is not possible to further reduce the V/III ratio to obtain a higher yield of A-polar NWs.

Changing the $\mathrm{V} / \mathrm{III}$ ratio strongly affects the contact angle of Ga droplets seated on top of developed NWs. Recently, contact angle engineering has been used to tune the growth direction and polarity of NWs. ${ }^{26,39}$ The contact angles and the growth interfaces of our A and B-polar GaAs NWs will be discussed shortly.

We now turn to the analysis of the polarity by AC-HAADF-STEM. Unless otherwise stated, the NWs considered here are covered with an $\mathrm{AlAs} / \mathrm{Al}_{0.33} \mathrm{Ga}_{0.67} \mathrm{As} / \mathrm{GaAs}$ shell heterostructure with nominal thicknesses of $10 \mathrm{~nm} /$ $100 \mathrm{~nm} / 10 \mathrm{~nm}$ respectively. $\mathrm{Al}_{0.33} \mathrm{Ga}_{0.67} \mathrm{As}$ shell is previously shown to contain self-assembled (Al)GaAs quantum dots $(\mathrm{QDs})^{40}$ whose optical properties are affected by the polarity. The AlAs and the final GaAs layers are used for enhancement of the QD formation ${ }^{50}$ and protection of $\mathrm{Al}_{0.33} \mathrm{Ga}_{0.67} \mathrm{As}$ against oxidation, respectively. Fig. 3(a) shows the low magnification STEM image of a NW. Fig. 3(b) shows atomically resolved AC-HAADF-STEM image of the middle part of the NW, demonstrating its defect-free crystal structure. Fig. 3(c) shows the profile intensity scan in the direction of the arrow shown in the inset of Fig. 3(b). This figure indicates that dumbbells are terminated by $\mathrm{Ga} / \mathrm{Al}$, confirming that this NW is A-polar. The crystal quality in the bulk of the NW is similar to the section shown in Fig. 3(b), except for the parts at the base which correspond to the initial stages of growth. Fig. 3(d) shows the neighboring sections of A, B and mixed polarities. The zoomed-in image of such sections is presented in Fig. 3(e). The atomically resolved detail of the mixed section is shown in Fig. 3(f), indicating 9R Ramsdell polytypism. ${ }^{41}$ The step in the (111) ortho-twin boundary that forms a coherent, six monolayers high $\Sigma=3(1-12)$ para-twin facet is also observed in Fig. $3(\mathrm{~g})$. The three monolayer step shows that the polarity across the $\{111\}$ plane is preserved on each side of the step and that the atomic arrangements on the opposite sides of the (1-12) facet are related by a mirror symmetry. The As atoms form an As-As bond, while the As and $\mathrm{Ga}$ atoms on the boundary plane become 5 -fold and 3 -fold coordinated, as shown previously by Sanchez et al. In this work, a very similar type of defect was observed in (111)B $\operatorname{GaAs}(\mathrm{P}) \mathrm{NWs}$. It was deemed to be detrimental to the functional properties of the structure, as it can act as a non-radiative recombination center. ${ }^{42}$ The NW base also presents twins perpendicular to the growth axis, as in Fig. $3(\mathrm{~g})$. These twins do not result in switching the polarity of the NW, which otherwise would imply the formation of an electrostatic potential barrier that scatters the carriers and reduces their mobility. ${ }^{15}$

The twins present at the base of the NW are in stark contrast with the defects shown in Fig. 3(h), in which we show the occurrence of lateral twins. ${ }^{43}$ These defects flip the polarity from A and B along the NW growth direction, but not across the twin boundary. This type of lateral twin is commonly observed in $\{111\}$ grown non-polar NWs (e.g., in Ga-catalyzed Si NWs). ${ }^{43}$ In our GaAs NWs, twins mostly occur along a B-polar direction, meaning that in a B-polar $\mathrm{NW}$, the twins observed will be perpendicular to the growth direction. In A-polar NWs, these twin boundaries will mainly occur along one of the three possible B-polar lateral facets, inclined $19^{\circ}$ with respect to the $<111>A$ direction. Furthermore, as the lateral twins are inclined with respect to the NW growth direction, although the polarity is always kept across the twin boundary, their presence implies a switch of polarity along the NW growth axis. Rarely, it is also observed that this sequence of defects can occur multiple times. As a general rule, we never observe twin defects perpendicular to the growth axis in A-polar NWs except in rare cases discussed below, demonstrating that the twinning tends to always occur along a B-polar direction. Based on the information given in Fig. 3, we conclude that A-polar NWs are almost defect-free along most of their length. In these regions, defects only occur along the lateral sides in rare cases, where the twinned region expands along the B direction versus the NW edge and quickly extinguishes. Formation of rotational or transversal twins perpendicular to the axis of A-polar NWs is even more rare, and is just observed at the root of the NW, where A and B-polar growth fronts are competing. The only observations of WZ phase in A-polar NWs are limited to the cases in which twins were formed, as each twin in ZB crystal is equivalent to a half monolayer of WZ phase. ${ }^{44} \mathrm{~A}$ continuous WZ section is never observed in A-polar NWs.

A closer look at the internal atomic structure of a B-polar NW is shown in Fig. 4. Fig. 4(a) shows the low magnification image of a NW. Fig. 4(b) and (c) give the atomically resolved crystal structure. The intensity profile scan along the arrow shown in Fig. 4(c) confirms that the dumbbells are terminated by As, showing that the NW is B-polar. Indeed, the presence of rotational/transversal twins, typical of B-polar GaAs NWs, is observed in Fig. 4(b) and (c). Unlike A-polar NWs, long WZ regions are easily observed in B-polar NWs, as depicted in Fig. 4(e). Switching of polarity to A is locally observed in just one occasion, shown in Fig. 4(f), in which the presence of a notch-like defect induces the formation of a lateral twin that propagates to the top of the NW. This creates a section of A-polar lateral growth on the NW side. Note that the polarity is always preserved across the twin boundary, but changes along the growth front on both sides of the twin boundary. This latter effect of a lateral twin defect propagating to the top of the NW with A-polarity, parallel to the main B-polarity of the $\mathrm{NW}$, is similar to the lateral twins that induce the formation of 

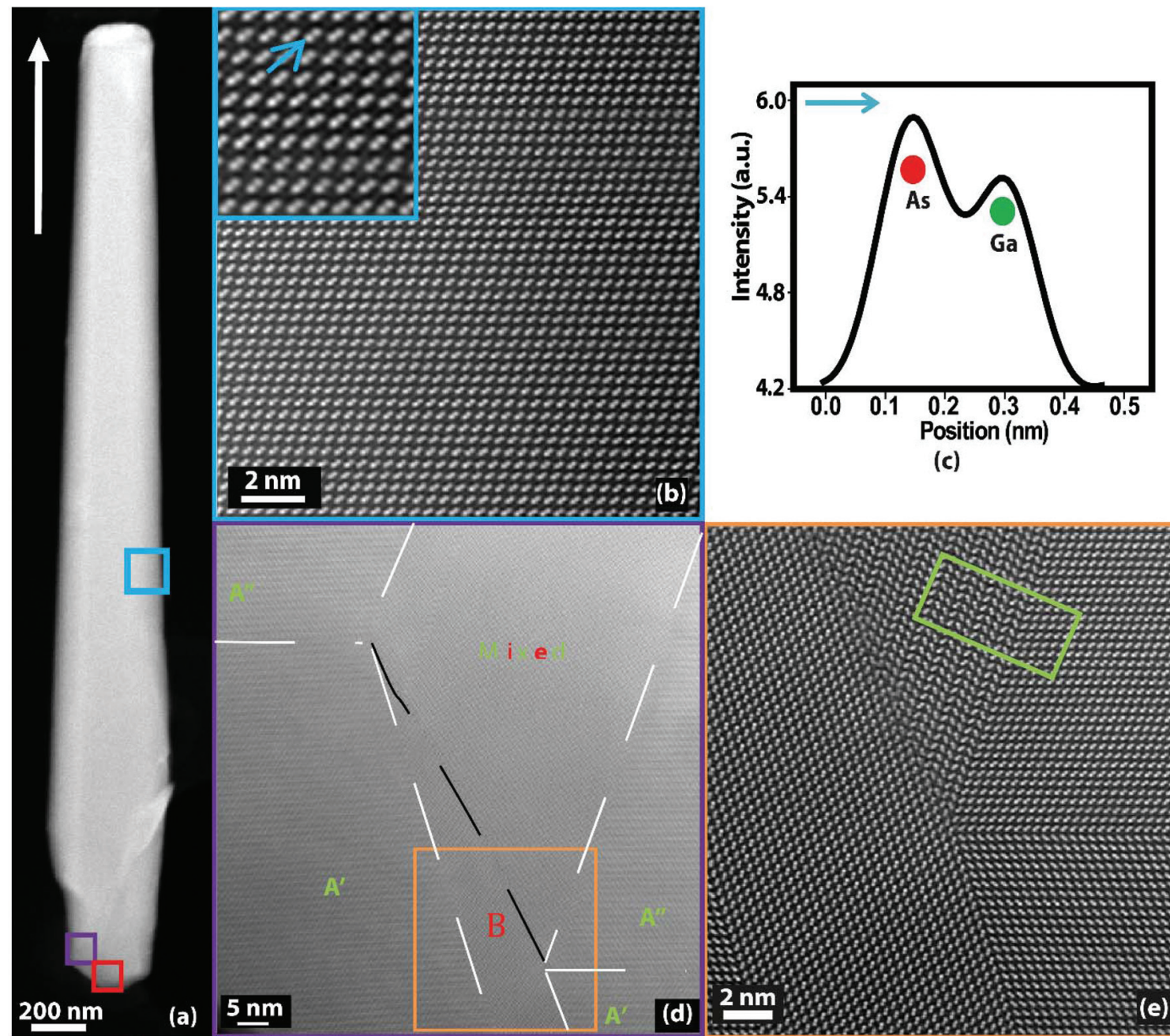

(c)
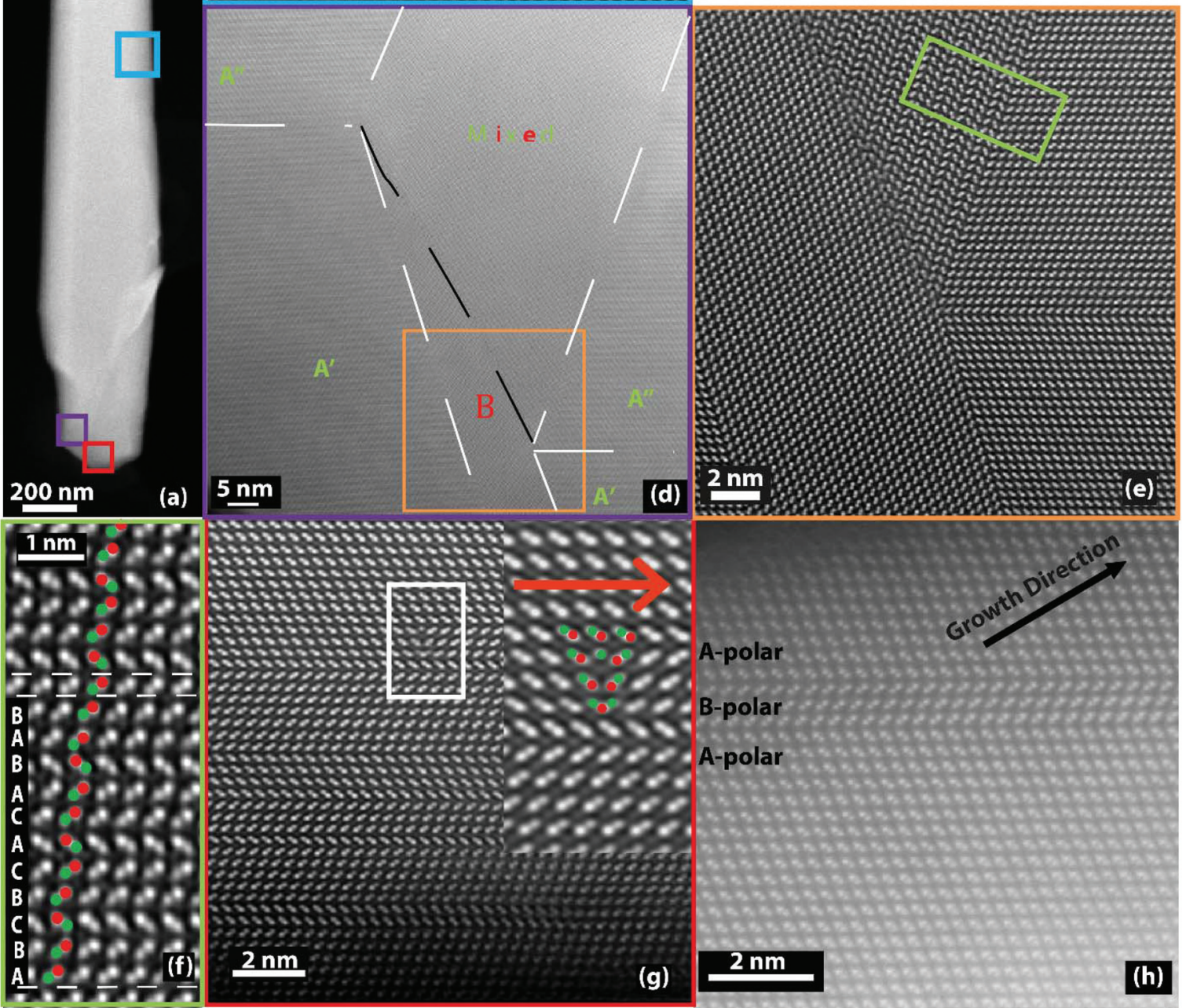

Fig. 3 Structural characterization of A-polar NWs. (a) Low magnification STEM image of an A-polar NW. The white arrow indicates the growth direction, which is the same for images in (a) to (g). (b) The crystal structure of the generic blue frame shown in (a). (c) Profile intensity scan in the direction of the arrow in (b), confirming the A-polar nature of the NW. (d) Competing regions of A and B-polar growths at the root of the NW. (e) Magnified image of the orange box in (d). (f) Magnified image of the green box shown in (e), showing 9R Ramsdell polytypism. (g) The presence of twins perpendicular to A-polar growth direction, and the details of a step in a (111) ortho-twin boundary; this defect is responsible for the in-plane polarity reversal. (h) The appearance of inclined twins in an A-polar NW. The black arrow shows the growth direction. Note that this image is from a different NW than the one shown in (a) to $(\mathrm{g})$. 

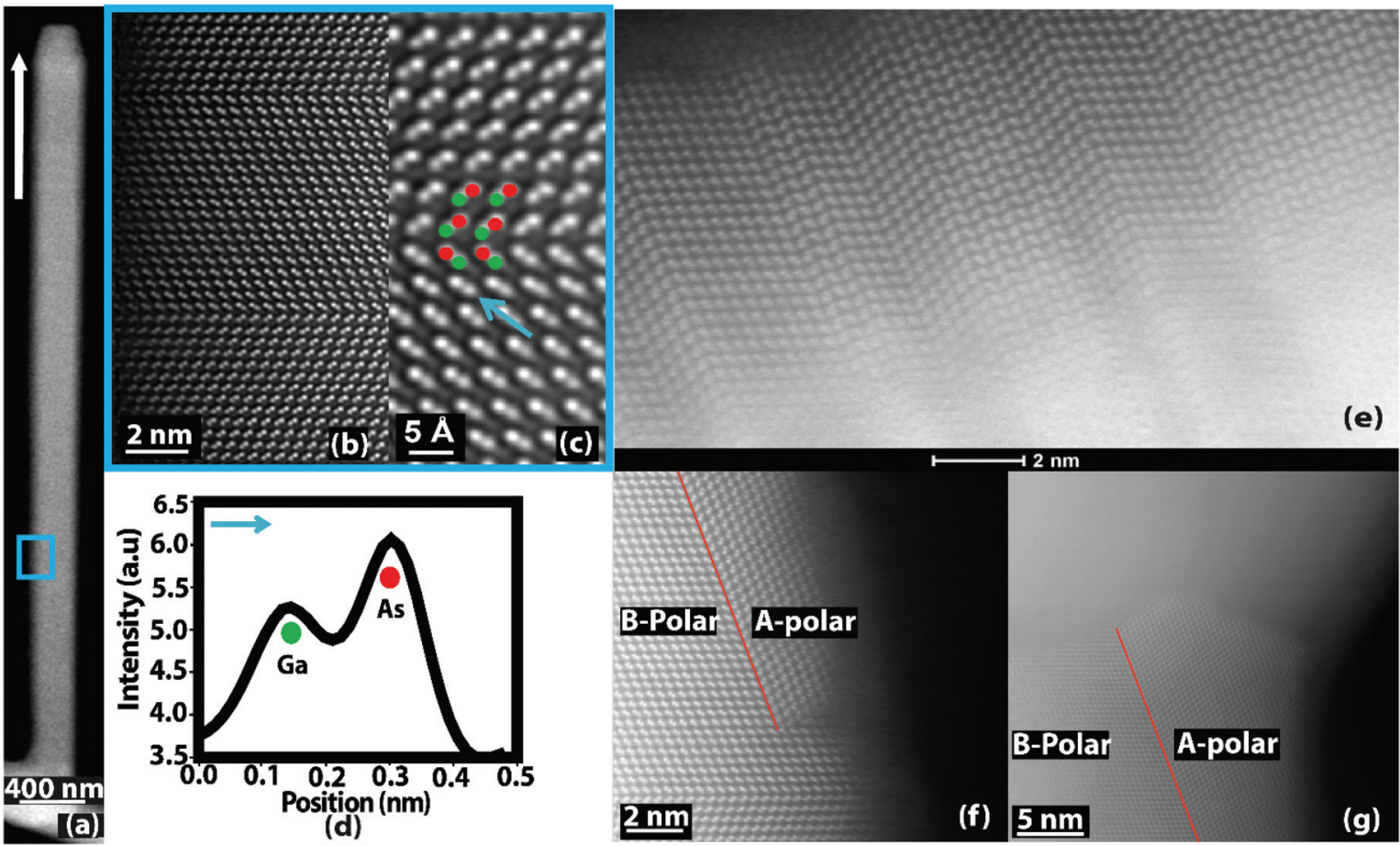

A-polar

(d)

(f) $5 \mathrm{~nm}$

(g)

Fig. 4 HAADF-STEM structural characterization of NWs with B-polarity. (a) Low magnification HAADF STEM image of a NW. (b) and (c) Atomically resolved details of the blue frame shown in (a), demonstrating the presence of rotational/transversal twins. (d) Profile intensity scan along the direction of the arrow shown in (c), confirming that the dumbbells are As-terminated and hence the NW is B-polar. (e) Image of a section of a B-polar NW, showing the occurrence of twins and WZ segments. (f) The occurrence of a notch-like defect, initiating a polarity reversal to the A-polar direction on the right, as opposed to the normal B-polar growth on the left of the image. (g) Configuration of the NW shown in ( $f$ ) under the droplet. Note that the images in (a) to (d), (e), and (f) to (g) are taken from different NWs.

nanosail-like InSb structures on top of InAs NW stems. ${ }^{18}$ Fig. 4(g) depicts the configuration of the neighbouring A and B-polar sections under the Ga droplet. The A-polar section originates at the notch-like defect found below. In short, unlike in the A-polar case, B-polar NWs show a high density of transversal twins and alternating sections of $\mathrm{ZB} / \mathrm{WZ}$ phases. In addition, B-polar NWs very rarely show the formation of notchlike defects that alter the polarity to A by nucleating a lateral twin.

To elaborate on the structural and morphological differences of A and B-polar NWs, we refer to Fig. 5. As mentioned previously, our A-polar NWs have an exceptionally clean and defect-free structure. This point is illustrated in Fig. 5(a), in which, except for the areas close to the base, the generic A-polar NW shows defect-free, single-crystalline ZB structure. A reconstruction of this $\mathrm{NW}$ obtained by stitching multiple HRTEM images is given in the ESI, Fig. SI1. $\dagger$ No twinning is observed in the body of this NW.

The presence of a region of competing and mixed polarities has important implications on the morphology of A-polar NWs. Comparing Fig. 3(a) and 4(a), one can see that A-polar NWs are tapered, as opposed to the B-polar NWs. This tapering is also apparent in Fig. 5(a). The detailed description of the cross-sectional evolution of A-polar NWs is presented in the ESI. $\dagger$ In short, after the polarity is stabilized to A, the NW has a hexagonal cross section that is stretched along one of its diagonals and hence is deviated from regular hexagonal shape. This geometry entails that the droplet has an ellipsoidal shape instead of a spherical shape. As growth continues, the droplet tries to adopt the energetically preferred spherical shape. ${ }^{37}$ This transition to a spherical geometry drives the cross section to the regular hexagonal shape. We believe that the observed tapering is caused by this transition.

For B-polar NWs, we observe that the body of the NW is composed of twinned $\mathrm{ZB}$ phase with inclusions of $\mathrm{WZ}$ phase. Excluding the exceptional cases such as the one depicted in Fig. 4(f) and (g), B-polar NWs obtained under these conditions are always terminated by the WZ segment, which is compatible with the previous observations. ${ }^{45,46}$ The cooling down process after growth is carried out under the As flux in order to prevent the decomposition of GaAs at the high growth temperature. $^{47,48}$ This effectively imposes an increased V/III ratio during the growth termination, which favors WZ phase formation. ${ }^{49,50}$

For gold-catalyzed GaAs NWs, it has been previously reported that A-polar NWs have higher contact angles compared to their B-polar counterparts. ${ }^{26}$ However, we did not observe any significant difference in the contact angles of $\mathrm{A}$ and B-polar NWs. We define here the contact angle $\beta$ between the droplet surface at the corner of the nanowire and 


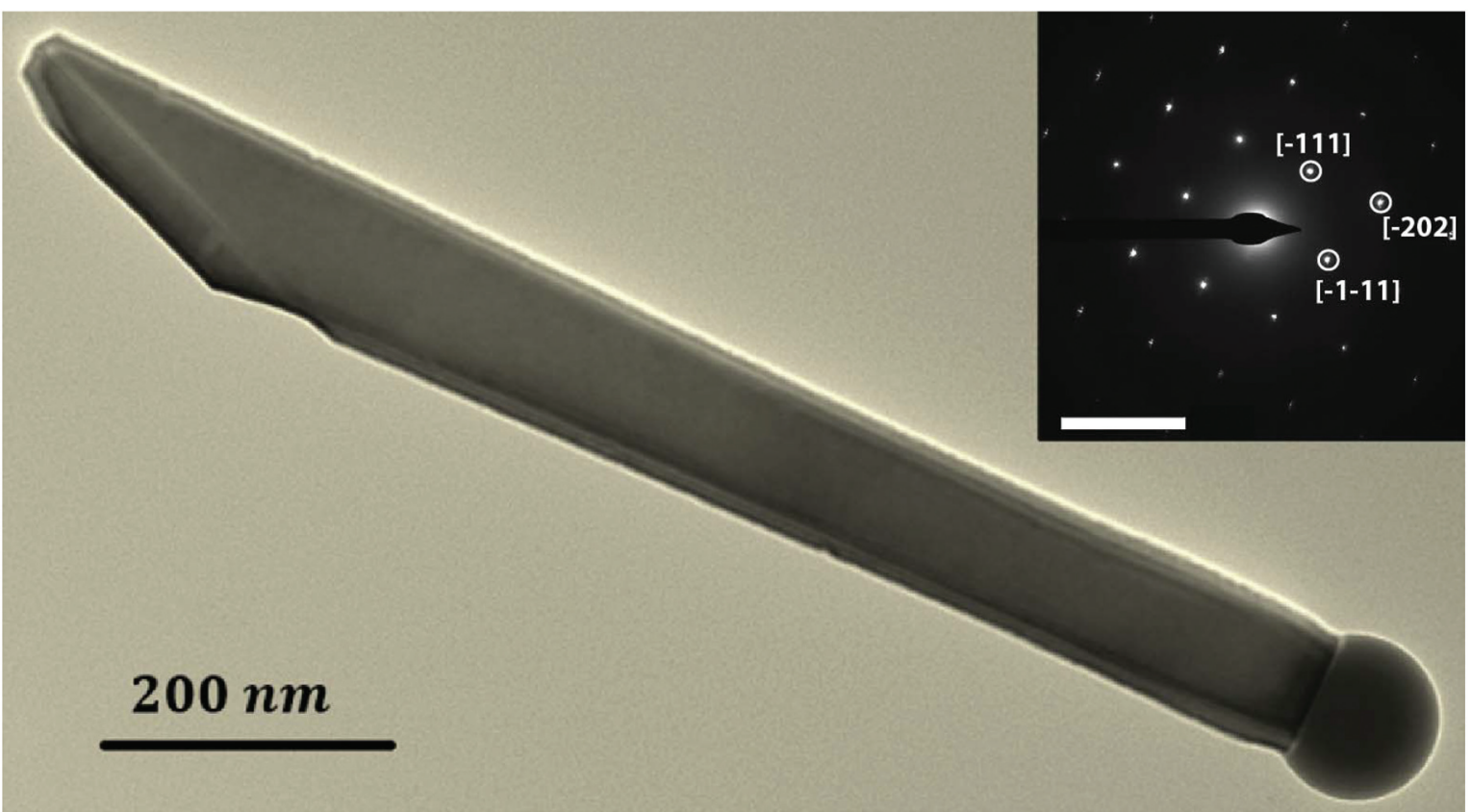

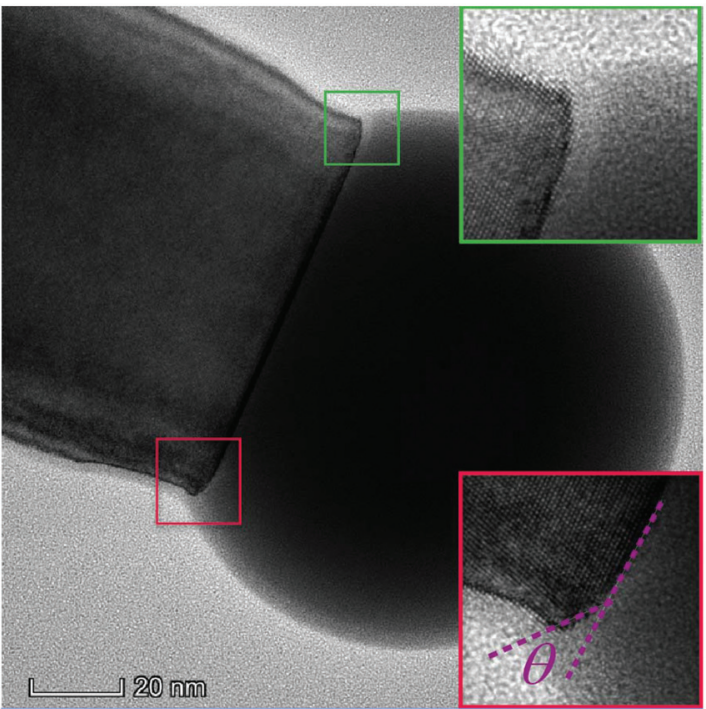

(b)

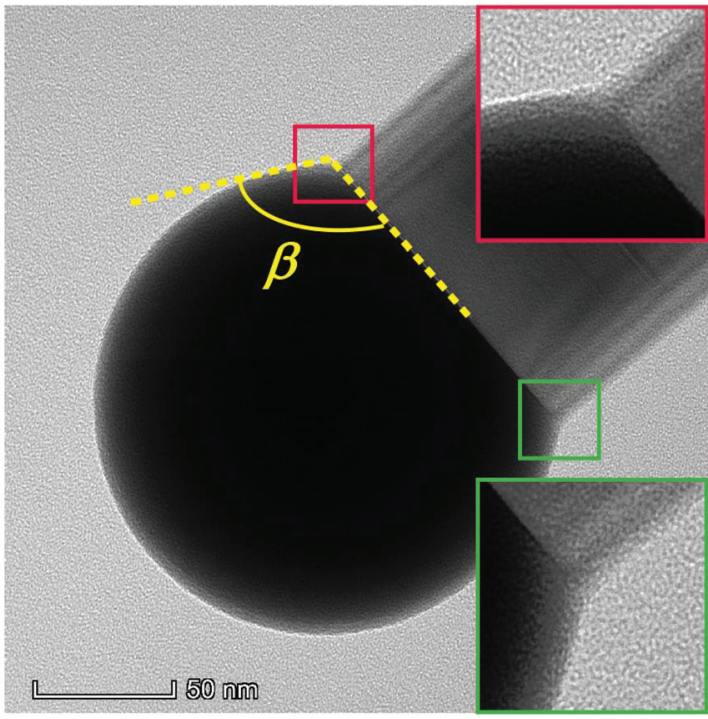

(c)

Fig. 5 (a) Low magnification bright field TEM image of an A-polar NW. The inset shows the diffraction pattern corresponding to the ZB phase, with the scale bar in the inset being $51 \mathrm{~nm}^{-1}$. (b) The droplet configuration of the A-polar NW shown in (a). The red and green insets depict the truncated growth front. (c) The droplet configuration of a B-polar NW, in which the growth front is always planar. These images are taken in [101] zone axis. The NWs depicted in (a), (b) and (c) are grown at a V/III ratio of 4.6 and a 2D-equivalent growth rate of $0.27 \AA \mathrm{s}^{-1}$; the SEM image of as-grown sample is presented in Fig. SI4(a). $\uparrow$ Note that the slight roughening of the NW sidewalls in (a) is caused by gradual oxidation of the GaAs, and is not a product of the growth process itself.

the overall growth surface (see sketch in Fig. 5). It does not consider the existence of a truncation. These values are obtained by ex situ measurements. The detailed explanation about the reason for having similar contact angles in A and B-polar NWs is presented in the ESI. $\dagger$

The exceptional crystal quality of A-polar NWs, with the effective absence of the WZ phase or twinning defects, calls for discussion. To understand the reason for the observed struc- tural difference of A and B-polar NWs, we compare the geometries of their growth interfaces. The images shown in Fig. 5(b) and (c) reveal the truncated growth front in A-polar NWs, with almost symmetrical inward tapered edge facets that are wetted by the droplet. Conversely, the growth front of B-polar NWs is always planar, and vertical sidewalls of such NWs are not wetted by the droplet. According to the current view, ${ }^{50,51}$ the truncation develops when the surface energy change of 
forming the truncation, $\Delta \gamma$, is negative, while positive $\Delta \gamma$ yields the planar growth interface. This $\Delta \gamma$ has the form

$$
\Delta \gamma=\frac{\gamma_{\theta \mathrm{L}}}{\cos \theta}-\gamma_{\mathrm{SL}} \tan \theta-\gamma_{\mathrm{OV}}+\gamma_{\mathrm{LV}} \sin \beta .
$$

According to this expression, the surface energy change contains the solid-liquid energy of the created inward tapered facet $\gamma_{\theta \mathrm{L}} / \cos \theta$ making the angle $\theta$ to the NW axis (see sketch in Fig. 5 for $\theta$ ), the increased area of the liquid-vapor interface $\gamma_{\mathrm{LV}} / \sin \beta$ and the two surface energies of the straight solidvapor facet parallel to the NW axis $\left(-\gamma_{0 \mathrm{~V}}\right)$ and the (111) solidliquid top facet $\left(-\gamma_{\mathrm{SL}} \tan \theta\right)$ eliminated by truncation. As mentioned above, the contact angles of the gallium droplets on top of A and B-polar NWs are very similar, which is why the last term in the equation can be considered identical. The side facets of both types of NWs, as well as the inward tapered facets, should also have very close atomic arrangements. Therefore, the main difference may originate from the different energies of the (111)A and (111)B top facets in contact with liquid, where the energy of the (111)A facet is expected to be larger according to the earlier discussion. This explains why, even for the same contact angles, the $-\gamma_{\mathrm{SL}} \tan \theta$ term favors the truncation in A-polar NWs and suppresses it in B-polar NWs.

Whenever the truncation is present, it cannot grow infinitely according to ref. 50, but rather adopts the energetically preferred size which oscillates in synchronization with the monolayer growth cycle. The droplet sliding down along the straight sidewalls is energetically suppressed. ${ }^{36}$ Therefore, the droplet is stable on top of the truncated A-polar NW. If all NW facets are truncated, as suggested by the image in Fig. 5(b), nucleation of two-dimensional islands does not occur at the triple phase line (TPL), but rather in the center of the liquidsolid interface. ${ }^{36,51}$ No WZ or twin formation is expected in this nucleation regime, because the islands are stable against either $60^{\circ}$ or $30^{\circ}$ rotation (producing the WZ phase or twins, respectively). In contrast to A-polar NWs, the island nucleation in B-polar NWs occurs at the TPL. In this case, the WZ islands or twinning defects emerge with a certain probability which depends on the chemical potential. ${ }^{23,26}$ High chemical potentials would favor predominantly WZ phase. ${ }^{36}$ However, due to the low V/III ratios employed in our growths, the chemical potential values are quite low, which explains the random occurrence of the WZ phase or twins in our B-polar NWs. This picture is fully consistent with all of our experimental observations and correlates with the results of ref. 49, where the truncated growth front in Ga-assisted GaAs NWs was also observed at low V/III ratios. The increase in the population of A-polar NWs for low V/III ratios can be explained by the fact that the truncated growth fronts that are required for the growth of these wires are more favored to be formed in these $\mathrm{V} / \mathrm{III}$ ratios.

Finally, we demonstrate the functionality of A-polar NWs by characterizing their optical properties. We measured the light emission of the NWs by CL at $20 \mathrm{~K}$ directly on an as-grown sample, and also on NWs dispersed on TEM grids. As stated above, the NWs were capped by the $\mathrm{AlAs} / \mathrm{Al}_{0.33} \mathrm{Ga}_{0.66} \mathrm{As} / \mathrm{GaAs}$ structure, yielding the formation of optically active (Al)GaAs QDs. We now correlate their occurrence and brightness with the polarity of the host NW. ${ }^{40}$

Fig. 6 presents the typical CL measurements. Fig. 6(a) shows the SEM micrograph of the investigated region, with the two polarities indicated by the corresponding arrows. Fig. 6(b) shows the panchromatic image of the CL signal, indicating that NWs with A-polarity have much more uniform emission intensity. Fig. 6(c) shows the emission mapping, color-coded into the three main emission wavelengths: 822, 836 and $854 \mathrm{~nm}$, corresponding 1.51, 1.48 and $1.45 \mathrm{eV}$, respectively. The lowest wavelength corresponds to the free exciton emission of GaAs at $20 \mathrm{~K}$, while the longer wavelengths can be attributed to the carbon contamination and polytypism. ${ }^{24}$ Between 820 and $860 \mathrm{~nm}$, A-polar NWs show homogeneous emission.

We now analyze the emission spectra of the NWs. Fig. 6(e-h) show representative emission spectra in the $1.41-1.53 \mathrm{eV}$ and 1.77-1.9 eV ranges. They were acquired from the two NWs with A and B polarities, with diameters of around $260 \mathrm{~nm}$. For each NW, spectra 1 to 3 correspond to excitation along the NW at three different points from the base to the top. We first focus on the first emission range, corresponding to the GaAs core emission. Considering an A-polar NW, the spectrum is clearly formed by two peaks, centered at $1.51 \mathrm{eV}$ and $1.48 \mathrm{eV}$. For this particular NW, the low-energy peak increases in intensity toward the NW top. The spectra of B-polar NWs are much broader and red-shifted with respect to the free exciton emission of GaAs.

To understand this drastically different optical behavior of the cores of A and B-polar NWs, we note that planar defects, such as stacking faults and twins, effectively introduce unitary $\mathrm{WZ}$ sections in the $\mathrm{ZB}$ phase (and vice versa). The $\mathrm{ZB} / \mathrm{WZ}$ GaAs heterostructure are known to have staggered type-II band alignment, ${ }^{52-55}$ which localizes electrons in the ZB and holes in the WZ segments. As a result, the core emission redshifts, which has been previously shown to depend on the density of defects. ${ }^{46}$ There is almost no intensity at $1.51 \mathrm{eV}$ for B-polar NWs, as expected for polytypic structures. $^{24}$ Furthermore, while the stacking faults and twins can act as effective traps for the carriers moving axially in the NWs, they allow for charge transfer perpendicular to the NW axis. ${ }^{56}$ The radial transport of the trapped carriers can broaden the excitonic emission by a fluctuating Stark effect. $^{56,57}$ In addition, by limiting the charge transport to the radial direction, these defects deplete the shell from excitons by favoring their transfer into the core, according to the gradient of the band gap between the core and the shell. ${ }^{58}$ In summary, the effects of structural defects on the core emission of B-polar NWs with respect to defect-free A-polar NWs manifest in (i) spectral broadening of the emission, (ii) an increased emission intensity of the core, and (iii) redshift of the emission. The narrower blue-shifted emission lines from A-polar NWs are compatible with their higher crystal quality. 


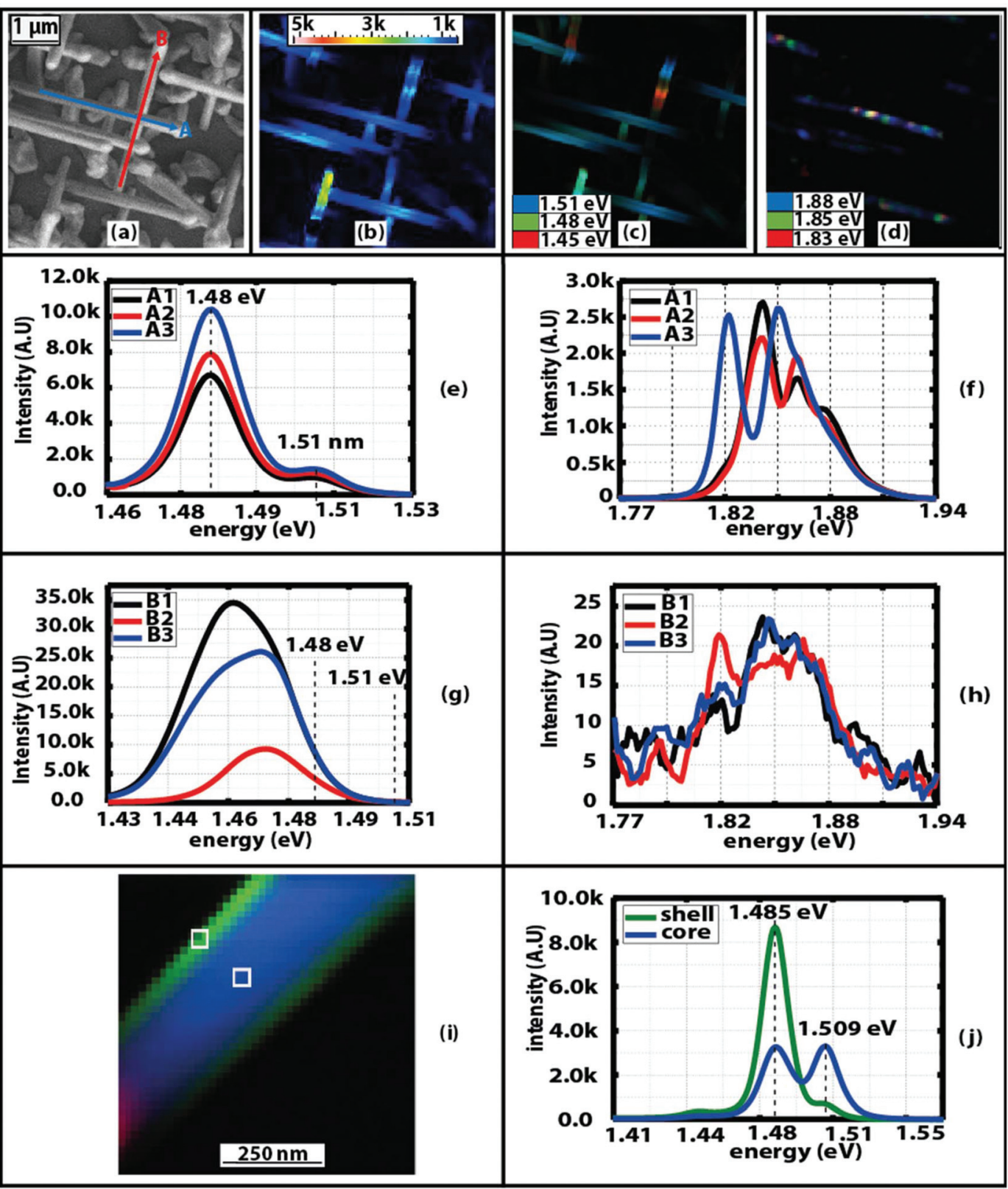

Fig. 6 Characterization of the optical properties of NWs with A and B-polarities using CL at $20 \mathrm{~K}$. (a) Top view SEM image captured inside the CL microscope. The arrows indicate the directions of the two different polarities. (b) Panchromatic intensity map for the identical image frame. (c) and (d) The wavelength-dependent $C L$ intensity map of the same region. (e) and (f) CL emission spectra collected from the three points of an A-polar NW, in the ranges 1.46-1.53 eV and 1.77-1.94 eV. The two ranges correspond to the emission from the GaAs core and the AlGaAs shell, respectively. (g) and (h) CL emission spectra collected from the three points of a B-polar NW (i) high-magnification wavelength-dependent map obtained on an A-polar NW lying on a TEM grid. The color code is the same as in (c). (j): CL spectra collected from the points indicated in the NW core and shell.

We now address the existence of a clear low-energy peak in the spectra of A-polar GaAs NWs at $1.48 \mathrm{eV}$. In order to ensure a better spatial resolution for observing the two peaks, we investigated the NWs on a TEM grid. Fig. 6(i) maps the emission at $1.51 \mathrm{eV}, 1.48 \mathrm{eV}$, and $1.44 \mathrm{eV}$. The intensities are normalized for clear determination of the different regions. We observe that the free exciton emission at $1.51 \mathrm{eV}$ is detected mainly when exciting the NWs core. The excitation map in the external regions of the NW shows two emission peaks at $1.48 \mathrm{eV}$ and $1.51 \mathrm{eV}$. We note the peak at 1.48 is more prominent. The emission at $1.48 \mathrm{eV}$ has previously been attributed to carbon. ${ }^{59-62}$ We believe that the carbon incorporation at the NW surface can occur during the CL investigation. We also note that a weak emission at $1.48 \mathrm{eV}$ is also observable upon excitation of the NW core, which should be due to diffusion and recombination of some excitons in the shell.

Emission at longer wavelengths can be observed only in the NW top. This can be attributed to fluctuations in the growth 
chamber at the very end of the growth, leading to morphological instabilities.

Finally, we study the second emission range (1.77-1.94 eV), which corresponds to the emission from the AlGaAs shell, as a function of the core polarity. It has previously been shown that compositional fluctuations in AlGaAs result in the formation of QDs that emit in the range between $1.77 \mathrm{eV}$ and $1.94 \mathrm{eV}$ $\mathrm{nm}^{40,63}$ In CL, these QDs appear as spatially localized emitters. ${ }^{40,63}$ Fig. 6(d) compares the CL mapping of A and B-polar NWs in the QD spectral region. Under these excitation conditions, we can find many spots of localized emission for most of A-polar NWs, while there is no significant emission from B-polar NWs. Representative emission spectra of A and B-polar NWs are compared in Fig. 6(f) and (h). The QD-like emission around $1.85 \mathrm{eV}$ is observed in both cases, with the emission of A-polar NWs being much brighter. As discussed earlier, defects in B-polar NWs act as the shortcuts for injecting excitons from the shell to the core. This migration depletes the QDs from excitons in B-polar NWs. The much lower density of defects in A-polar NWs means that the excitons remain in the QDs, which is why the QD emission in these NWs shows two orders of magnitude higher intensity with respect to B-polar NWs. This enhancement is observable in Fig. 6(d), (f) and (h).

\section{Conclusions}

In conclusion, we have reported an optimized growth of A-polar GaAs NW on GaAs(100) substrates by the Ga-assisted VLS method. Decreasing the V/III flux ratio has been found crucial for improving the yield of A-polar NWs up to 57\%. Similar to the Au-catalyzed VLS method, A-polar GaAs NWs are generally free of stacking defects along their entire length, while B-polar NWs suffer from random twining and WZ insertions. Exceptional crystal quality of A-polar NWs is understood through the truncated geometry of their growth interface, which suppresses the TPL nucleation and hence the formation of stacking faults. We speculate that the truncation develops in A-polar NWs due to the higher surface energy of the top (111)A facet with respect to the (111)B one. We have correlated the optical properties of the NWs with their polarity. Luminescence from A-polar NWs is homogeneous along the NW, with an intense peak around $1.51 \mathrm{eV}$ $(820 \mathrm{~nm})$. This peak corresponds to the free exciton in ZB GaAs, in agreement with the higher crystal quality of A-polar NWs. The effect of crystal quality of the host NW on the emission properties of AlGaAs QDs in the shell has been shown for the first time. The QD emission from the AlGaAs shells is two orders of magnitude brighter in A-polar NWs. This is explained by a larger number of excitons in QDs surrounding such NWs. This opens a clear path toward the fabrication of bright QDs in GaAs, which have many promising applications in optoelectronic devices.

This work opens many novel fundamental questions in the current understanding and state-of-the-art of nanowire growth as well as new avenues for crystal-phase and growth direction engineering.

\section{Conflicts of interest}

There are no conflicts to declare.

\section{Acknowledgements}

The funding for this research was provided by Swiss National Science Foundation (SNSF), National Centre of Competence in Research Quantum Science and Technology (NCCR QSIT) and Horizon 2020 European Union Funding for Research and Innovation through projects INDEED and LIMQUET. The authors express their gratitude to the staff of EPFL's Interdisciplinary Center for Electron Microscopy (CIME), particularly Dr Davide Deiana, for the support. SMS acknowledges funding from "Programa Internacional de Becas "la Caixa"Severo Ochoa". SMS and JA acknowledge funding from Generalitat de Catalunya 2017 SGR 327 and the Spanish MINECO ENE2017-85087-C3-3-R project. ICN2 acknowledges support from the Severo Ochoa Program (MINECO, Grant SEV-2013-0295) and is funded by the CERCA Programme/ Generalitat de Catalunya. Part of the present work has been performed in the framework of Universitat Autònoma de Barcelona Materials Science $\mathrm{PhD}$ program. Part of the HAADF-STEM microscopy was conducted in the Laboratorio de Microscopias Avanzadas at the Instituto de Nanociencia de Aragon-Universidad de Zaragoza. JA and SMS thank them for offering access to their instruments and expertise. This work has received funding from the European Union's Horizon 2020 Research and Innovation Programme under grant agreement No. 654360 NFFA-Europe. VGD thanks the Russian Foundation for Basic Research for financial support under grants 17-5216017 , 16-29-03129, 16-02-00134, 16-29-03283, and 18-0240006 .

\section{Notes and references}

1 R. S. Wagner and W. C. Ellis, Appl. Phys. Lett., 1964, 4, 8990.

2 M. Takahasi, M. Kozu and T. Sasaki, Jpn. J. Appl. Phys., 2016, 55, 04EJ04.

3 Z. H. Wu, X. Y. Mei, D. Kim, M. Blumin and H. E. Ruda, Appl. Phys. Lett., 2002, 81, 5177-5179.

4 P. Schroth, J. Jakob, L. Feigl, S. M. Mostafavi Kashani, J. Vogel, J. Strempfer, T. F. Keller, U. Pietsch and T. Baumbach, Nano Lett., 2018, 18, 101-108.

5 M. Takahasi, M. Kozu, T. Sasaki and W. Hu, Cryst. Growth Des., 2015, 15, 4979-4985.

6 D. V. Lang, H. G. Grimmeiss, E. Meijer and M. Jaros, Phys. Rev. B: Condens. Matter Mater. Phys., 1980, 22, 3917-3934.

7 J. Tersoff, Nano Lett., 2015, 15, 6609-6613. 
8 P. Krogstrup, R. Popovitz-Biro, E. Johnson, M. H. Madsen, J. Nygård and H. Shtrikman, Nano Lett., 2010, 10, 44754482.

9 B. Mandl, J. Stangl, E. Hilner, A. A. Zakharov, K. Hillerich, A. W. Dey, L. Samuelson, G. Bauer, K. Deppert and A. Mikkelsen, Nano Lett., 2010, 10, 4443-4449.

10 S. J. Gibson, J. P. Boulanger and R. R. LaPierre, Semicond. Sci. Technol., 2013, 28, 105025.

11 B. Bauer, A. Rudolph, M. Soda, A. Fontcuberta i Morral, J. Zweck, D. Schuh and E. Reiger, Nanotechnology, 2010, 21, 435601.

12 S. Ambrosini, M. Fanetti, V. Grillo, A. Franciosi and S. Rubini, J. Appl. Phys., 2011, 109, 094306.

13 C. Colombo, D. Spirkoska, M. Frimmer, G. Abstreiter and A. Fontcuberta i Morral, Phys. Rev. B: Condens. Matter Mater. Phys., 2008, 77, 155326.

14 A. Fontcuberta i Morral, C. Colombo, G. Abstreiter, J. Arbiol and J. R. Morante, Appl. Phys. Lett., 2008, 92, 063112.

15 M. de la Mata, C. Magen, J. Gazquez, M. I. B. Utama, M. Heiss, S. Lopatin, F. Furtmayr, C. J. Fernández-Rojas, B. Peng, J. R. Morante, R. Rurali, M. Eickhoff, A. Fontcuberta i Morral, Q. Xiong and J. Arbiol, Nano Lett., 2012, 12, 2579-2586.

16 F. Schuster, M. Hetzl, S. Weiszer, J. A. Garrido, M. de la Mata, C. Magen, J. Arbiol and M. Stutzmann, Nano Lett., 2015, 15, 1773-1779.

17 M. I. B. Utama, F. J. Belarre, C. Magen, B. Peng, J. Arbiol and Q. Xiong, Nano Lett., 2012, 12, 2146-2152.

18 M. de la Mata, R. Leturcq, S. R. Plissard, C. Rolland, C. Magén, J. Arbiol and P. Caroff, Nano Lett., 2016, 16, 825833.

19 M. de la Mata, C. Magén, P. Caroff and J. Arbiol, Nano Lett., 2014, 14, 6614-6620.

20 F. Schuster, B. Laumer, R. R. Zamani, C. Magén, J. R. Morante, J. Arbiol and M. Stutzmann, ACS Nano, 2014, 8, 4376-4384.

21 M. I. B. Utama, M. de la Mata, C. Magen, J. Arbiol and Q. Xiong, Adv. Funct. Mater., 2013, 23, 1636-1646.

22 H. Potts, Y. van Hees, G. Tütüncüoglu, M. Friedl, J.-B. Leran and A. Fontcuberta i Morral, Cryst. Growth Des., 2017, 17, 3596-3605.

23 F. Glas, J.-C. Harmand and G. Patriarche, Phys. Rev. Lett., 2007, 99, 146101.

24 D. Spirkoska, J. Arbiol, A. Gustafsson, S. Conesa-Boj, F. Glas, I. Zardo, M. Heigoldt, M. H. Gass, A. L. Bleloch, S. Estrade, M. Kaniber, J. Rossler, F. Peiro, J. R. Morante, G. Abstreiter, L. Samuelson and A. Fontcuberta i Morral, Phys. Rev. B: Condens. Matter Mater. Phys., 2009, 80, 245325.

25 I. Zardo, S. Conesa-Boj, F. Peiro, J. R. Morante, J. Arbiol, E. Uccelli, G. Abstreiter and A. Fontcuberta i Morral, Phys. Rev. B: Condens. Matter Mater. Phys., 2009, 80, 245324.

26 X. Yuan, P. Caroff, J. Wong-Leung, L. Fu, H. H. Tan and C. Jagadish, Adv. Mater., 2015, 27, 6096-6103.

27 I. Forrest, M. Davidson, D. C. Lee, A. Dayne, D. Fanfair and B. A. Korgel, J. Phys. Chem. C, 2007, 111, 2929-2935.
28 R. R. Zamani, S. Gorji Ghalamestani, J. Niu, N. Sköld and K. A. Dick, Nanoscale, 2017, 9, 3159-3168.

29 A. Fontcuberta i Morral, J. Arbiol, J. D. Prades, A. Cirera and J. R. Morante, Adv. Mater., 2007, 19, 1347-1351.

30 K. W. Schwarz and J. Tersoff, Nano Lett., 2011, 11, 316-320.

31 Y. Yuan and T. R. Lee, in Surface Sciecne Techniques, 2013, pp. 3-34.

32 F. Matteini, G. Tütüncüoglu, D. Mikulik, J. VukajlovicPlestina, H. Potts, J.-B. Leran, W. C. Carter and A. Fontcuberta i Morral, J. Cryst. Growth, 2016, 16, 57815786.

33 F. Matteini, G. Tütüncüoğlu, D. Rüffer, E. Alarcón-Lladó and A. Fontcuberta i Morral, J. Cryst. Growth, 2014, 404, 246-255.

34 F. Matteini, G. Tütüncüoglu, D. Mikulik, J. VukajlovicPlestina, H. Potts, J.-B. Leran, W. C. Carter and A. Fontcuberta i Morral, Cryst. Growth Des., 2016, 16, 57815786.

35 F. Matteini, G. Tütüncüoglu, H. Potts, F. Jabeen and A. Fontcuberta i Morral, Cryst. Growth Des., 2015, 15, 31053109.

36 V. G. Dubrovskii, Cryst. Growth Des., 2017, 17, 2589-2593.

37 J. Berthier and K. A. Brakke, The physics of microdroplets, John Wiley \& Sons, Inc, 2012.

38 N. Moll, A. Kley, E. Pehlke and M. Scheffler, Phys. Rev. B: Condens. Matter Mater. Phys., 1996, 54, 8844-8855.

39 J. Wang, S. R. Plissard, M. A. Verheijen, L.-F. Feiner, A. Cavalli and E. P. A. M. Bakkers, Nano Lett., 2013, 13, 3802-3806.

40 M. Heiss, Y. Fontana, A. Gustafsson, G. Wüst, C. Magen, D. D. O'Regan, J. W. Luo, B. Ketterer, S. Conesa-Boj, A. V. Kuhlmann, J. Houel, E. Russo-Averchi, J. R. Morante, M. Cantoni, N. Marzari, J. Arbiol, A. Zunger, R. J. Warburton and A. Fontcuberta i Morral, Nat. Mater., 2013, 12, 439-444.

41 L. S. Ramsdell, Am. Mineral., 1945, 32, 64-82.

42 A. M. Sanchez, Y. Zhang, E. W. Tait, N. D. M. Hine, H. Liu and R. Beanland, Nano Lett., 2017, 17, 2454-2459.

43 S. Conesa-Boj, I. Zardo, S. Estradé, L. Wei, P. Jean Alet, P. Roca i Cabarrocas, J. R. Morante, F. Peiró, A. F. i Morral and J. Arbiol, Cryst. Growth Des., 2010, 10, 1534-1543.

44 J. Arbiol, A. Fontcuberta i Morral, S. Estradé, F. Peiró, B. Kalache, P. Roca i Cabarrocas and J. R. Morante, J. Appl. Phys., 2008, 104, 064312.

45 F. Jabeen, V. Grillo, S. Rubini and F. Martelli, Nanotechnology, 2008, 19, 275711.

46 M. Heiss, S. Conesa-Boj, J. Ren, H.-H. Tseng, A. Gali, A. Rudolph, E. Uccelli, F. Peiró, J. R. Morante, D. Schuh, E. Reiger, E. Kaxiras, J. Arbiol and A. Fontcuberta i Morral, Phys. Rev. B: Condens. Matter Mater. Phys., 2011, 83, 045303.

47 T. E. Haynes, W. K. Chu, T. L. Aselage and S. T. Picraux, Appl. Phys. Lett., 1986, 49, 666-668.

48 J. Tersoff, D. E. Jesson and W. X. Tang, Phys. Rev. Lett., 2010, 105, 035702.

49 W. Kim, V. G. Dubrovskii, J. Vukajlovic-Plestina, G. Tütüncüoglu, L. Francaviglia, L. Güniat, H. Potts, 
M. Friedl, J.-B. Leran and A. Fontcuberta i Morral, Nano Lett., 2018, 18, 49-57.

50 D. Jacobsson, F. Panciera, J. Tersoff, M. C. Reuter, S. Lehmann, S. Hofmann, K. A. Dick and F. M. Ross, Nature, 2016, 531, 317-322.

51 C.-Y. Wen, J. Tersoff, K. Hillerich, M. C. Reuter, J. H. Park, S. Kodambaka, E. A. Stach and F. M. Ross, Phys. Rev. Lett., 2011, 107, 025503.

52 P. Krogstrup, M. Hannibal Madsen, W. Hu, M. Kozu, Y. Nakata, J. Nygård, M. Takahasi and R. Feidenhans'l, Appl. Phys. Lett., 2012, 100, 093103.

53 P. Kusch, E. Grelich, C. Somaschini, E. Luna, M. Ramsteiner, L. Geelhaar, H. Riechert and S. Reich, Phys. Rev. B: Condens. Matter Mater. Phys., 2014, 89, 045310.

54 L. Ahtapodov, H. Kauko, A. M. Munshi, B. O. Fimland, A. T. J. van Helvoort and H. Weman, J. Appl. Phys., 2017, 122, 245102.

55 D. Spirkoska, A. L. Efros, W. R. L. Lambrecht, T. Cheiwchanchamnangij, A. Fontcuberta i Morral and G. Abstreiter, Phys. Rev. B: Condens. Matter Mater. Phys., 2012, 85, 045309.
56 D. Dalacu, K. Mnaymneh, J. Lapointe, X. Wu, P. J. Poole, G. Bulgarini, V. Zwiller and M. E. Reimer, Nano Lett., 2012, 12, 5919-5923.

57 G. Sallen, A. Tribu, T. Aichele, R. André, L. Besombes, C. Bougerol, S. Tatarenko, K. Kheng and J. P. Poizat, Phys. Rev. B: Condens. Matter Mater. Phys., 2009, 80, 085310.

58 J. Nelson, The Physics Of Solar Cells.

59 G. Tütüncüoglu, The growth and optical properties of III-V nanostructures grown by Molecular Beam Epitaxy, EPFL, 2017.

60 Hannah Jane Joyce, Growth and Characterisation of III-V Semiconductor Nanowires for Optoelectronic Device Applications, Australian National University, 2009.

61 B. J. Skromme and G. E. Stillman, Phys. Rev. B: Condens. Matter Mater. Phys., 1984, 29, 1982-1992.

62 B. J. Skromme, T. S. Low, T. J. Roth, G. E. Stillman, J. K. Kennedy and J. K. Abrokwah, J. Electron. Mater., 1983, 12, 433-457.

63 L. Francaviglia, Y. Fontana, S. Conesa-Boj, G. Tütüncüoglu, L. Duchêne, M. B. Tanasescu, F. Matteini and A. Fontcuberta i Morral, Appl. Phys. Lett., 2015, 107, 033106. 